\title{
BMJ Open Demographic risk factors for COVID-19 infection, severity, ICU admission and death: a meta-analysis of 59 studies
} Bart G Pijls (1) , ${ }^{1}$ Shahab Jolani, ${ }^{2}$ Anique Atherley, ${ }^{3}$ Raissa T Derckx, ${ }^{4}$
Janna I R Dijkstra, ${ }^{5}$ Gregor H L Franssen, ${ }^{6}$ Stevie Hendriks, ${ }^{7}$ Anke Richters
Annemarie Venemans-Jellema,,${ }^{9}$ Saurabh Zalpuri, ${ }^{10}$ Maurice P Zeegers ${ }^{11}$
To cite: Pijls BG, Jolani S, Atherley A, et al. Demographic risk factors for COVID-19 infection, severity, ICU admission and death: a meta-analysis of 59 studies. BMJ Open 2021;11:e044640. doi:10.1136/ bmjopen-2020-044640

- Prepublication history and supplemental material for this paper is available online. To view these files, please visit the journal online (http://dx.doi. org/10.1136/bmjopen-2020044640).

Received 08 September 2020 Revised 16 November 2020 Accepted 14 December 2020

Check for updates

(C) Author(s) (or their employer(s)) 2021. Re-use permitted under CC BY-NC. No commercial re-use. See rights and permissions. Published by BMJ.

For numbered affiliations see end of article.

Correspondence to

Dr Bart G Pijls;

b.g.c.w.pijls@lumc.nl

\section{ABSTRACT}

Objective We aimed to describe the associations of age and sex with the risk of COVID-19 in different severity stages ranging from infection to death.

Design Systematic review and meta-analysis.

Data sources PubMed and Embase through 4 May 2020. Study selection We considered cohort and case-control studies that evaluated differences in age and sex on the risk of COVID-19 infection, disease severity, intensive care unit (ICU) admission and death.

Data extraction and synthesis We screened and included studies using standardised electronic data extraction forms and we pooled data from published studies and data acquired by contacting authors using random effects meta-analysis. We assessed the risk of bias using the Newcastle-Ottawa Scale.

Results We screened 11.550 titles and included 59 studies comprising 36.470 patients in the analyses. The methodological quality of the included papers was high (8.2 out of 9). Men had a higher risk for infection with COVID-19 than women (relative risk (RR) $1.08,95 \% \mathrm{Cl}$ 1.03 to 1.12 ). When infected, they also had a higher risk for severe COVID-19 disease (RR $1.18,95 \% \mathrm{Cl} 1.10$ to 1.27), a higher need for intensive care (RR $1.38,95 \% \mathrm{Cl}$ 1.09 to 1.74 ) and a higher risk of death (RR $1.50,95 \% \mathrm{Cl}$ 1.18 to 1.91 ). The analyses also showed that patients aged 70 years and above have a higher infection risk (RR 1.65, $95 \% \mathrm{Cl} 1.50$ to 1.81 ), a higher risk for severe COVID-19 disease (RR 2.05, 95\% $\mathrm{Cl} 1.27$ to 3.32), a higher need for intensive care (RR 2.70, 95\% Cl 1.59 to 4.60 ) and a higher risk of death once infected ( $\mathrm{RR} 3.61,95 \% \mathrm{Cl} 2.70$ to 4.84 ) compared with patients younger than 70 years.

Conclusions Meta-analyses on 59 studies comprising 36.470 patients showed that men and patients aged 70 and above have a higher risk for COVID-19 infection, severe disease, ICU admission and death.

PROSPERO registration number CRD42020180085.

\section{BACKGROUND}

COVID-19 or the disease caused by the SARS-CoV-2 coronavirus has caused a pandemic that has affected patients in more than 188 countries and territories around the world. The number of patients diagnosed with COVID-19 has exceeded 27 million on 8
Strengths and limitations of this study

- Our search strategy revealed 11.550 individual records and we included 59 studies.

- Our study focuses on the early phase of the pandemic.

- A thorough sensitivity analysis could not refute the conclusions.

- Our review has added a quality assessment of the individual studies.

- Most included studies, $n=50$, were from China involving Chinese patients with COVID-19 compared with $n=9$ studies from outside China.

September 2020, and to date more than 890 000 patients have died. ${ }^{1}$

Regarding demographics, respiratory tract infections are, in general, more severe in men and they tend to lead to higher mortality in men. ${ }^{2}$ Higher mortality for men was also observed during the severe acute respiratory syndrome (SARS) epidemic. ${ }^{3}$ In a mixed group of patients with COVID-19 and SARS, Jin et at found that increased age and sex were associated with more severe disease and mortality. However, a systematic review on the association between demographic factors and different severity stages of COVID-19 is lacking.

Knowledge on the association between demographic factors and different severity stages of COVID-19 such as infection, severe disease, intensive care unit (ICU) admission and death may provide insight into the underlying pathophysiological mechanisms (immunity, coagulopathy and comorbidities). This knowledge may also guide clinical decisionmaking, especially when there is an impending shortage in healthcare resources such as ICU beds. Additionally, exploring demographic factors influencing COVID-19 outcomes may guide policymakers in, for instance, the prioritisation of non-pharmaceutical interventions 
and screening. ${ }^{5}$ These demographic factors may also be important for the design and interpretation of clinical trials on the efficacy of treatments as they could potentially be strong confounders. Therefore, the aim of this living systematic review is to describe the association between demographic factors and COVID-19 in different stages of the disease.

\section{METHODS}

The reporting of this living systematic review and metaanalysis is in accordance with the Preferred Reporting Items for Systematic Reviews and Meta-Analyses (PRISMA) statement and a protocol has been registered a priori at the PROSPERO registry (PROSPERO 2020). ${ }^{6}$ For this review, we focused on the early phase of the pandemic.

Demographic factors include: age, sex, social economic status (education level), pregnancy and ethnicity. As only a few studies so far reported on the latter three factors, the current version of this review focuses on age and sex. Age was categorised into old age, defined as 70 years and older, and young age, defined as younger than 70 years. Seventy years was chosen as a cut-off point for the main analyses because this was the most commonly used cut-off in the first studies included. We also collected data on other cut-off points (60 and 65 years) where possible. We considered four stages of disease severity: (1) infection, (2) severe clinical or radiological symptoms (according to WHO guidance ${ }^{7}$ ), (3) ICU admission, and (4) death. This led to the following research questions:

What is the association between demographic factors and:

1. A confirmed COVID-19 infection among the general population?

2. Clinically/radiologically severe COVID-19 among hospitalised patients with a confirmed infection?

3. ICU admission among patients hospitalised for confirmed COVID-19 infection?

4. Death among patients hospitalised for confirmed COVID-19 infection?

Originally, we also planned to investigate 'hospitalisation' as a potential outcome. However, only one study reported on this, which did not warrant inclusion in this version of the review. Future versions of the review will re-evaluate 'hospitalisation' as an outcome. The cases and controls for each stage of the disease are defined in table 1 .

\section{Data sources and searches}

The search strategy was devised with a specialised librarian (GHLF) and the following databases were searched from December 2019 up to 4 May 2020: Medline via PubMed and Embase. Additionally, EPPI Centre (COVID-19: a living systematic map of the evidence) was consulted up to 31 March $2020 .{ }^{8}$

We designed the search strategy to be sensitive and reproducible. The term COVID-19 was elaborated in combinations of controlled vocabulary and free text terms. See online supplemental appendix 1 for the full search strategy. No language restrictions were applied during the search strategy. Studies reported in languages spoken by the research team were included: English, Dutch, German, French and Russian. Studies published in any other language were temporarily excluded and will be reconsidered in future updates of this living review.

\section{Study selection}

Initial screening on the basis of title and abstract of eligible studies was performed by one reviewer (RTD, AVJ or BGP). A second reviewer (RTD) redid the study selection procedure on a random sample of 500 studies. The between-reviewer agreement from these 500 studies was $98.4 \%$ with a kappa of 0.74 , indicating substantial agreement. ${ }^{9}$ When the information in the abstract did not suffice or where there was any doubt, the studies remained potentially eligible. The full text of potentially eligible studies was independently evaluated in duplicate by two reviewers (from AR, SZ, AA, JIRD, SH). All records identified through the searches were collected in an electronic reference database and subjected to the following inclusion and exclusion criteria: the study had to focus on humans with COVID-19 or SARS-CoV-2 coronavirus infections providing, or potentially providing, sufficient information to calculate risk ratios for our prespecified associations (table 1). A study was excluded when no valid comparisons could be made. This was the case when less than five observations were reported in any cell of the contingency tables, when the study quality score (see next paragraph) was less than 5 out of 9 and when patients were admitted to hospital for different indications than for COVID-19 (eg, kidney transplant patients, patients with fractured bones).

\section{Table 1 Study structure}

\begin{tabular}{llll}
\hline Severity stage & Case & Control & Population \\
\hline 1. Infection & Test positive & Test negative & General population \\
2. Severe symptoms (clinically or radiologically) & Severe symptoms & Non-severe symptoms & Hospitalised COVID-19 cases \\
3. ICU admittance & Admitted to ICU & Not admitted to ICU & Hospitalised COVID-19 cases \\
4. Death & Death & Alive & Hospitalised COVID-19 cases \\
\hline
\end{tabular}

ICU, intensive care unit. 


\section{Data extraction and quality assessment}

Observed frequencies of outcomes and controls per level of the determinants were extracted from text, tables or figures (ie, $2 \times 2$ tables leading to unadjusted risk ratios) for each included study. One reviewer (AR or SZ) extracted data from included studies regarding the severity stages of COVID-19, patient demographics and study characteristics in a predefined electronic data sheet that was designed during a pilot data extraction phase on the first eligible studies. A second reviewer (AA, JIRD or $\mathrm{SH})$ double-checked the inclusion by the data extractors. Any disagreements were resolved by consensus or by consulting a referee (BGP or MPZ). We contacted the authors of papers with data presented in a way that did not allow summarisation in contingency tables by email. We sent a reminder email after 1 week. In total, we contacted 87 authors of whom 17 supplied additional data which could be used in the analyses for 12 papers. Risk of bias of the included studies was appraised independently by one reviewer (from AA, JIRD or SH) using the NewcastleOttawa Scale (NOS). ${ }^{10}$

\section{Data synthesis and analysis}

We used the relative risk (RR) to assess the association between each severity stage (ie, diagnosis, severe disease, ICU admission and death) and demographic factors. The data from the included studies underwent random effects meta-analysis to determine the pooled effect sizes with corresponding 95\% CIs and (in case of heterogeneity) $95 \%$ prediction intervals. ${ }^{11}$ The amount of statistical heterogeneity was assessed through visual inspection of the forest plots and by calculating $\mathrm{I}^{2}$ statistics. ${ }^{12}$ If data allowed, we explored potential sources of statistical heterogeneity when $\mathrm{I}^{2}$ was above $40 \%$ (1) through subgroup analyses and (2) with random effects meta-regression analyses on predefined factors. These factors include: geographical region, study quality, study size, days into the pandemic, publication date, diagnostic modality (eg, PCR test, CT signs, clinical symptoms and their combinations that led to the diagnosis of COVID-19) and clinical setting (eg, nursing home, home, hospital, general practitioner cohort). We carried out leaveone-out analyses to determine the influence of possible outlier studies on the pooled effect size. The study setting and diagnostic modality were very consistent within the different outcomes, so a sensitivity on these factors was not meaningful.

To assess publication bias we constructed funnel plots for visual inspection and statistically tested potential asymmetry using the Egger and Harbord test. ${ }^{13}{ }^{14}$ In case of asymmetry, a trim-and-fill method and cumulative metaanalyses were used to explore the magnitude and direction of publication bias.

\section{Patient and public involvement}

This systematic review and meta-analysis is part of the WHO Evidence Collaborative on COVID-19 answering their rapid review priority questions on risk factors for infection and disease severity. Patients were not involved.

\section{RESULTS}

\section{Study selection}

The literature search yielded 11550 unique hits of which 300 studies were eligible after screening titles and abstracts. From these eligible studies, we excluded 241: 13 were reviews; 17 were written in a language not spoken by the review team; 118 did not report or evaluate demographic factors; and 93 had no valid comparisons between cases and controls. This left 59 studies in the current metaanalysis, covering a total of 36470 patients. ${ }^{15-73}$ Details of the study selection are given in figure 1 (PRISMA flow chart).

\section{Study characteristics}

We included studies on the effect of age (70 years or more vs less than 70 years) and sex (men vs women). There were either no studies or not enough studies on social economic status, pregnancy or ethnicity to allow any meaningful analyses. Regarding age and sex, there were not enough studies on the outcome 'hospitalization' to allow any meaningful analyses. The current meta-analysis therefore presents results on age and sex regarding risk of infection, disease severity, ICU admission and death.

From the included studies, 50 were from China, 3 from the USA, 1 from Germany, 1 from Iran, 1 from Italy, 1 from Singapore, 1 from South Korea and 1 from the UK. The included studies were published between 2 January 2020 and 15 April 2020. The mean age of the patients in the included studies ranged from 7 to 73 years. The percentage of males in the included papers ranged from $35 \%$ to $81 \%$. The follow-up ranged from 12 to 73 days. For details of individual studies, organised by exposure and outcome, see online supplemental appendix 2.

\section{Risk of bias}

The methodological quality of the included papers was high with an average of 8.2 out of 9 , as measured with the NOS. Case definition and case representativeness were acceptable in 55 out of 59 and 55 out of 59 studies, respectively. Control selection and control definition were acceptable in 59 out of 59 and 55 out of 59 studies, respectively. Exposure ascertainment and comparable ascertainment were acceptable in 57 out of 59 and 58 out of 59 studies, respectively. Non-response rate was not applicable for our study questions. Details of NOS items for individual studies, organised by exposure and outcome, are available in online supplemental appendix 2.

\section{Synthesis of results}

Meta-analyses of the primary outcomes for the risk factors sex and age revealed differences among men and women and among patients 70 years of age or older $(70+)$ and below 70 years $(70-)$. An overview of the pooled results 


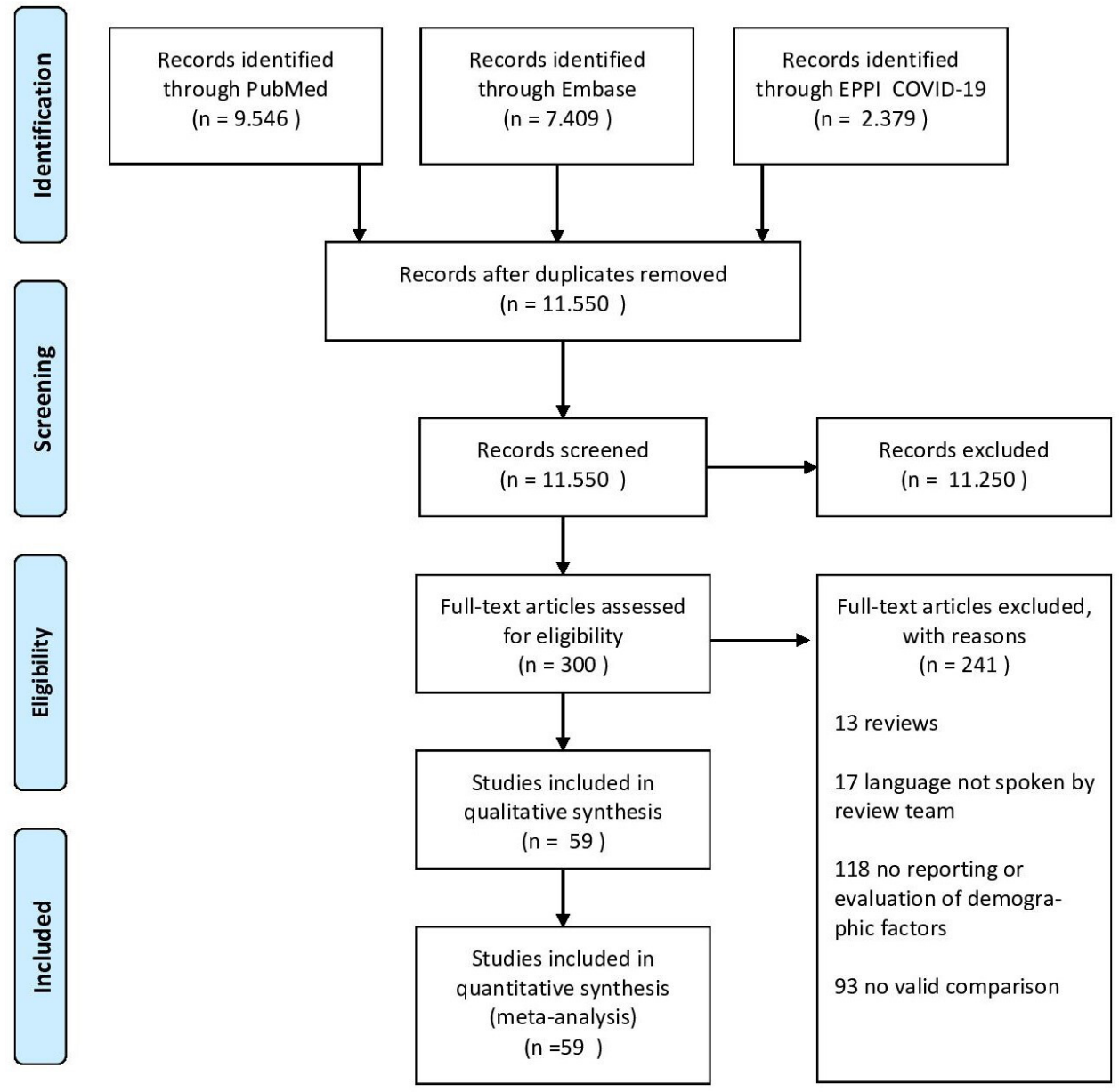

Figure 1 Preferred Reporting Items for Systematic Reviews and Meta-Analyses (PRISMA) flow chart showing study selection.

from random effects meta-analyses for each demographic factor separately can be found in table 2 .

\section{Demographic factor: sex}

There was an unambiguous association between each stage of disease severity and sex with men having a higher risk of infection, disease severity, ICU admission and death than women. Men have a statistically significant $8 \%$ higher risk of being diagnosed with COVID-19 than women (RR: 1.08 , 95\% CI 1.03 to $1.12 ; 8$ studies) (see figure 2). When diagnosed, men also experienced more severe disease than women (RR 1.18, 95\% CI 1.10 to $1.27 ; 35$ studies), implying that the risk of severe COVID-19 disease for men is $18 \%$ higher than that for women (see figure 3). Moreover, the rate of admission to ICU in patients with COVID-19 was higher among men as compared with women. The aggregated random effect was 1.38 (95\% CI 1.09 to $1.74 ; 11$ studies) (see figure 4 ). Finally, we observed that men were at higher risk of death

\begin{tabular}{|c|c|c|c|c|c|c|c|}
\hline Exposure & Outcome & Studies (n) & Patients (n) & $\begin{array}{l}\text { Pooled } \\
\text { estimate (RR) }\end{array}$ & $95 \% \mathrm{Cl}$ & 95\% PI & $\begin{array}{l}\text { Heterogeneity } \\
\left(\left(^{2}\right)\right.\end{array}$ \\
\hline \multirow{4}{*}{$\begin{array}{l}\text { Sex } \\
\text { (male vs female) }\end{array}$} & Infection & 8 & 16286 & 1.08 & 1.03 to 1.12 & NA & $0 \%$ \\
\hline & Severe disease & 35 & 7832 & 1.18 & 1.10 to 1.27 & NA & $15 \%$ \\
\hline & $\mathrm{ICU}$ & 11 & 1493 & 1.38 & 1.09 to 1.74 & NA & $32 \%$ \\
\hline & Death & 14 & 12792 & 1.50 & 1.18 to 1.91 & 0.73 to 3.10 & $62 \%$ \\
\hline \multirow{4}{*}{$\begin{array}{l}\text { Age } \\
(70+\text { vs } 70-)\end{array}$} & Infection & 4 & 12996 & 1.65 & 1.50 to 1.81 & NA & $35 \%$ \\
\hline & Severe disease & 7 & 1102 & 2.05 & 1.27 to 3.32 & 0.42 to 9.93 & $87 \%$ \\
\hline & ICU & 5 & 688 & 2.70 & 1.59 to 4.60 & 0.47 to 15.7 & $69 \%$ \\
\hline & Death & 5 & 9222 & 3.61 & 2.70 to 4.84 & 1.51 to 8.67 & $60 \%$ \\
\hline
\end{tabular}

ICU, intensive care unit; NA, not applicable; PI, prediction interval; RR, risk ratio. 
Relative risk of COVID-19 infection male vs female

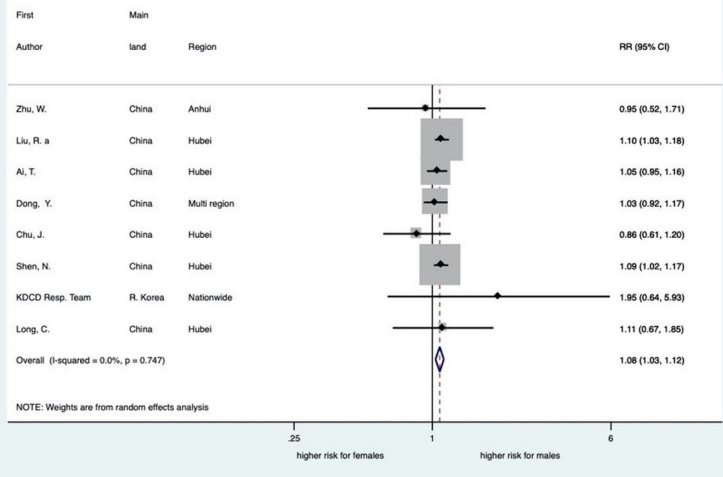

Figure 2 Forest plot showing the association between sex and risk of COVID-19 infection. Overall, men have a 1.08 times higher risk of COVID-19 infection than women. Liu et al. ${ }^{32} \mathrm{RR}$, relative risk.

from COVID-19 as compared with women (RR 1.50, 95\% CI 1.18 to $1.91 ; 14$ studies) (see figure 5 ). These increased risks for men across all severity stages were statistically significant, with little heterogeneity (see table 2).

\section{Demographic factor: age}

This meta-analysis also showed a clear-cut distinction between patients aged 70 years or older $(70+)$ and 70 years or younger $(70-)$ with respect to each stage of disease severity for COVID-19 (see figures 6-9). Patients aged $70+$ appear to have a $65 \%$ higher risk for infection of COVID-19 (RR 1.65, 95\% CI 1.50 to $1.81 ; 4$ studies).

\section{Relative risk of severe COVID-19 disease male vs female}

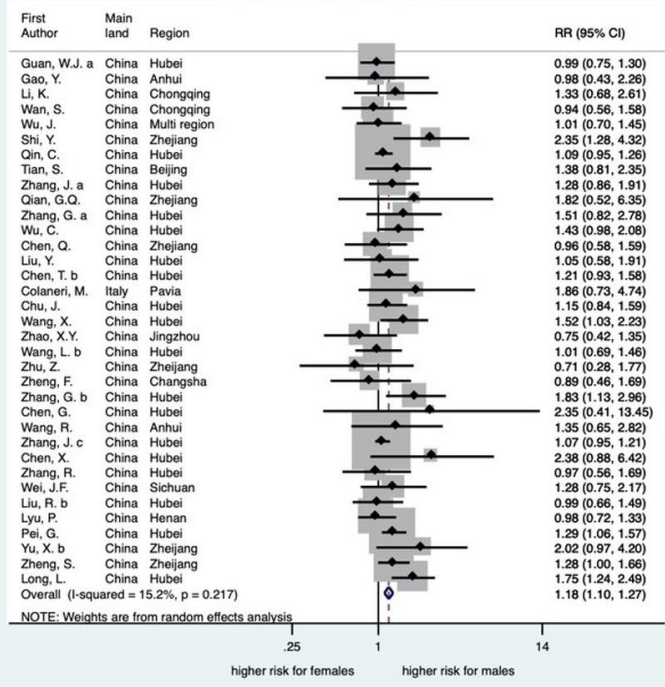

Figure 3 Forest plot showing the association between sex and risk of severe COVID-19. Overall, men have a 1.18 times higher risk of severe COVID-19 than women. Zhang et al; ${ }^{67}$ Zhang et $a l ;{ }^{65}$ Zhang et al; ${ }^{64}$ Zhang et al; ${ }^{66}$ Liu et al. ${ }^{33} \mathrm{RR}$, relative risk.
Relative risk of admission to ICU in COVID-19 patients male vs female

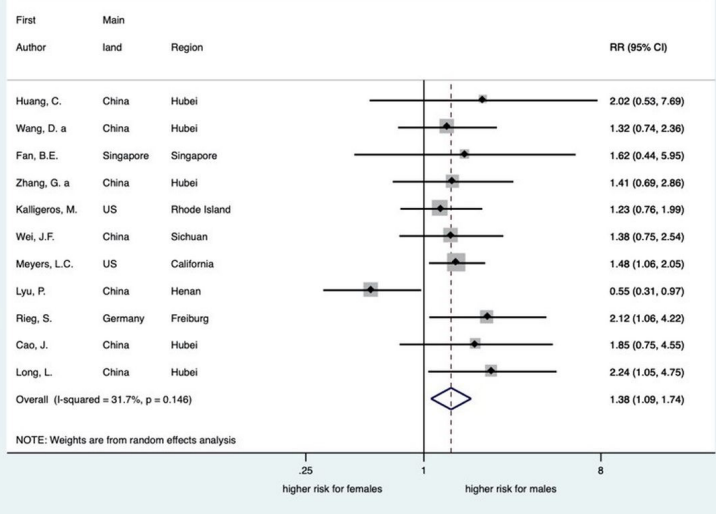

Figure 4 Forest plot showing the association between sex and risk of ICU admission due to COVID-19. Overall, men have a 1.38 times higher risk of ICU admission due to COVID-19 than women. Zhang et al. ${ }^{65} \mathrm{ICU}$, intensive care unit; $R R$, relative risk.

When infected, they also appear to have a higher risk for severe COVID-19 disease, need for intensive care and death (RR 2.05, 95\% CI 1.27 to 3.32; 7 studies, RR 2.70, 95\% CI 1.59 to $4.60 ; 5$ studies, and RR 3.61, 95\% CI 2.70 to $4.84 ; 5$ studies, respectively). These increased risks for older patients across all severity stages were statistically significant and very consistent, though there was some observed heterogeneity in the magnitude of this effect but not in the direction of the effect.

\section{Sensitivity analyses}

Funnel plots showed some asymmetry for the relation between sex and the outcomes of severe disease, ICU admission and death (all $\mathrm{p}$ values above 0.063; Harbord test). Although the subsequent trim-and-fill

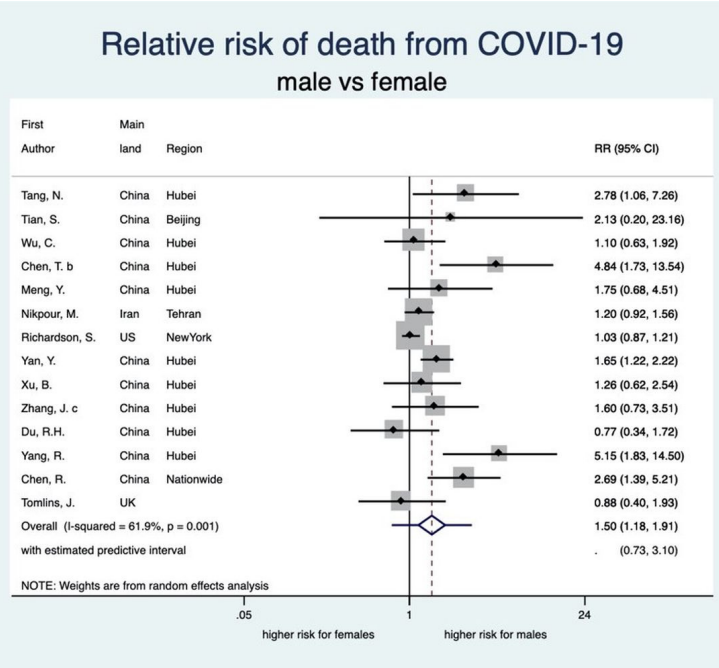

Figure 5 Forest plot showing the association between sex and risk of death due to COVID-19. Overall, men have a 1.50 times higher risk of death due to COVID-19 than women. RR, relative risk. 
Relative risk of COVID-19 infection $70+$ versus 70 -

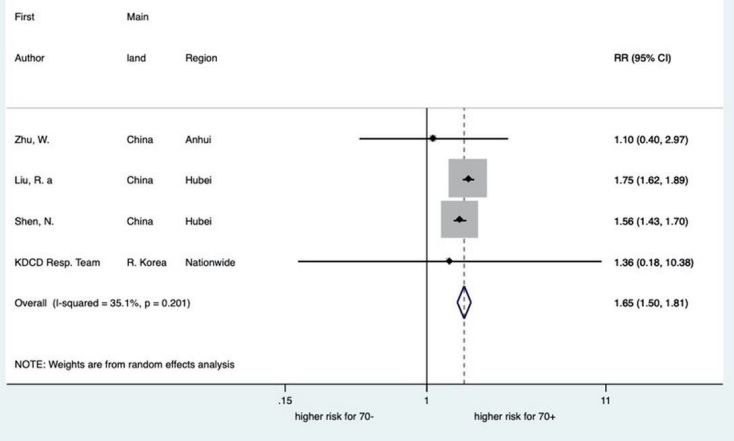

Figure 6 Forest plot showing the association between age and risk of COVID-19 infection. Overall, patients aged 70 years or older have a 1.65 times higher risk of COVID-19 infection than patients younger than 70 years. Liu et al. ${ }^{32} \mathrm{RR}$, relative risk.

analysis revealed some reduction in the effect sizes, all conclusions remained the same. More specifically, the RR for severity changed from 1.18 to 1.16 , for ICU from 1.38 to 1.20 and for death from 1.50 to 1.20 . We also redid the meta-analysis by excluding studies with possible overlap in patients, to make sure each patient was only included once. We assumed this to be the case when studies were similar in terms of region, recruitment period and hospital; in a group of studies with a possible overlap, only the largest study was included in the analysis. The results remained almost identical (see table 3). We also performed exhaustive sensitivity analyses consisting of subgroup analyses and meta-regression (see online supplemental appendix 3). The conclusions of our study did not change in subgroups, nor were any factors identified as significant sources of heterogeneity in meta-regression analyses. The main reason for this is the low level between study variance. For sex, however,

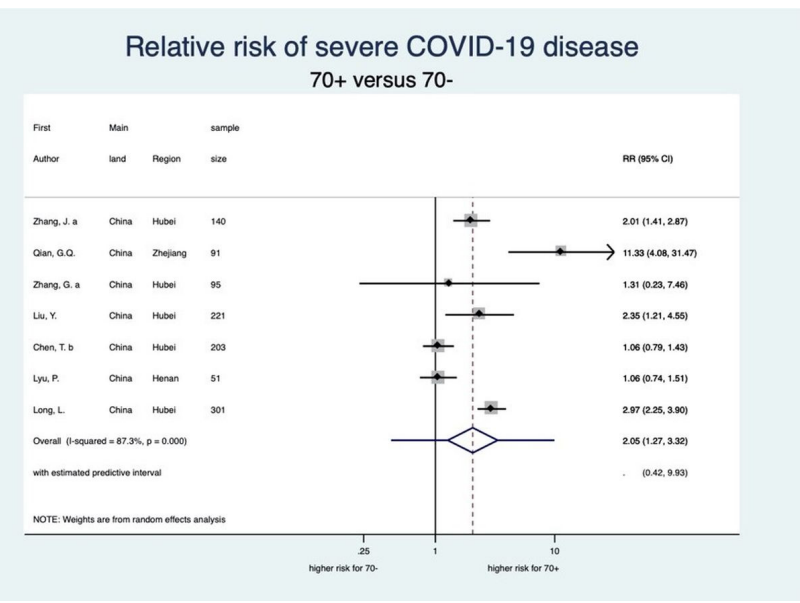

Figure 7 Forest plot showing the association between age and risk of severe COVID-19. Overall, patients aged 70 years or older have a 2.05 times higher risk of severe COVID-19 than patients younger than 70 years. Zhang et $a / ;{ }^{67}$ Zhang et al. ${ }^{65} \mathrm{RR}$, relative risk.
Relative risk of admission to ICU in COVID-19 patients $70+$ versus 70

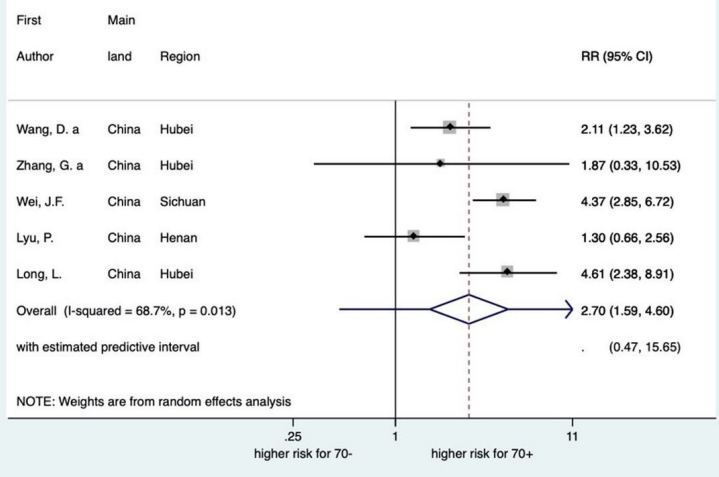

Figure 8 Forest plot showing the association between age and risk of ICU admission due to COVID-19. Overall, patients aged 70 years or older have a 2.70 times higher risk of ICU admission due to COVID-19 than patients younger than 70 years. Zhang et al. ${ }^{65} \mathrm{ICU}$, intensive care unit; RR, relative risk.

little heterogeneity was observed. For age, there was some heterogeneity in the magnitude of this effect but not in the direction of the effect.

\section{DISCUSSION}

\section{Summary of evidence}

In this systematic review we described the association between demographic factors and COVID-19 infection, severity, ICU admission and death. There were not enough data to report on pregnancy, socioeconomic status or ethnicity. Our results showed that men were more often severely affected by COVID-19 than women on all stages of the disease. Men more often had a higher risk for COVID-19 infection. When hospitalised with COVID-19, men more often developed severe COVID-19 disease and more often required intensive care admission, ultimately

Relative risk of death from COVID-19 $70+$ versus 70

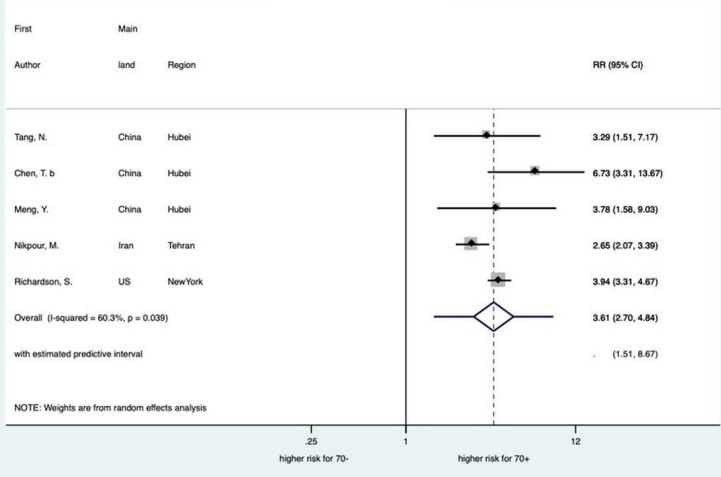

Figure 9 Forest plot showing the association between age and risk of death due to COVID-19. Overall, patients aged 70 years or older have a 3.61 times higher risk of death due to COVID-19 than patients younger than 70 years. RR, relative risk. 
Table 3 Exclusion of possible overlaps

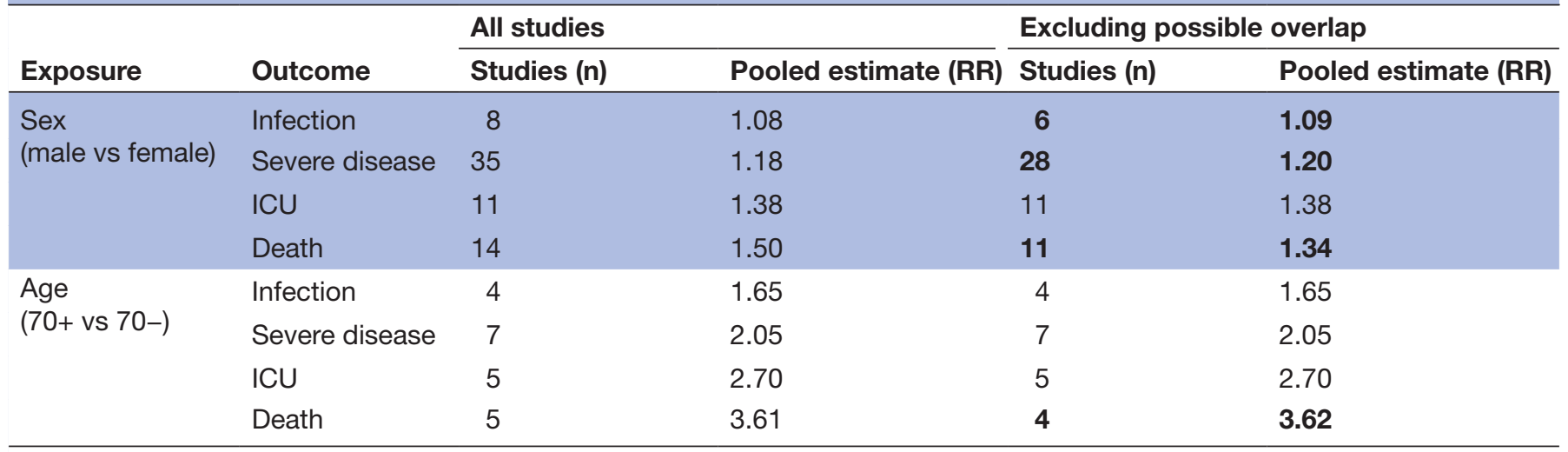

Studies with possible overlap of patients were excluded from the analysis, results presented in bold. Possible overlap was assumed when studies were from the same region, recruitment period and hospital. In a group of studies with possible overlap only the largest study was included in the analysis. The results remained almost identical.

$\mathrm{ICU}$, intensive care unit; RR, risk ratio.

resulting in death more often. We also found that patients aged 70 years and above affected by COVID-19 were more often observed to have confirmed COVID infection, severe disease, ICU admission and dying compared with patients younger than 70 years.

A living systematic review design was chosen because during the COVID-19 pandemic there is an urgent need for the most up-to-date evidence while maintaining scientific rigour and quality. ${ }^{74}$ Additionally, studies relevant for these research questions will likely be continuously published in the foreseeable future. Moreover, traditional systematic reviews risk becoming rapidly outdated when new evidence is published almost on a daily basis, and it is not an option to wait until the pandemic is over to publish a systematic review on the full body of evidence. ${ }^{7677}$

\section{Possible explanations}

This study looked at unadjusted risk ratios for the demographic factors age and sex for several COVID outcomes. Although some studies have reported adjusted risk ratios, this indicates a different goal. Adjustment is only relevant when attempting to look at causal effects, in which case the causal effect will be validly estimated after full adjustment for all confounders, while simultaneously avoiding adjustment for colliders and mediating factors. Given that the optimal adjustment factors are not yet known and also differ across various research questions, settings and, most importantly, across time and place, we consider this undesirable. For the purpose of the current study, unadjusted risk ratios were considered most appropriate.

This observation of higher risk of severe disease and higher risk of dying for men compared with women when affected by COVID-19 is in line with the fact that, in general, respiratory tract infectious diseases are more severe in men and subsequently tend to lead to higher mortality in men. ${ }^{2}$ Moreover, during the SARS epidemic of 2003, mortality was also higher in men. ${ }^{3}$ Thus, this increased severity of respiratory tract disease, including COVID-19, and increased mortality for men may point to an underlying biological mechanism. Aside from anatomical, lifestyle, behavioural, comorbidities and socioeconomic differences between men and women it has been suggested that differences in the immune system between men and women may, at least, partially explain the observed sex differences in the incidence and severity of respiratory tract infections. ${ }^{2}$ Indeed, several groups have found sex differences in the immune response, including the innate immune response. ${ }^{78} 79$ Regarding COVID-19, there are indications that immune response (inflammation) markers such as interleukin-6 (IL-6) are associated with severity and mortality. ${ }^{80}$ In a broader perspective, immune response markers, such as IL-6, have also been associated with worse outcome and higher mortality in trauma patients. ${ }^{82} 83$ Thus, in addition to differences in health and comorbidities between men and women, differences in the way the immune system responds to the COVID-19 infection may also play a role in the pathogenesis and the outcome of the disease.

Similar to sex differences in immune response, the immune system also changes with age. Ageing is, among others, characterised by a chronic proinflammatory status of the immune system with persistent low-grade innate immune activation that may increase tissue damage caused by infections in the elderly. ${ }^{84} 85$ Ageing is also associated with a high prevalence of comorbidities and decreased reserve capacity of vital organs which may lead to increased frailty, and together with an aged immune system this may put elderly individuals at risk of a poor outcome and higher risk of mortality when infected with COVID-19.

\section{Implications for clinicians, policymakers and researchers}

Regardless of the underlying mechanism, the observed demographic differences in COVID-19 severity may contribute by informing clinical and policy guidelines in the prioritisation of non-pharmaceutical interventions and screening for COVID-19 in groups at risk of worse outcome. The observation that men and patients aged 70 
years and above have a higher risk of severe disease, ICU admission and death when infected with COVID-19 may guide individual clinical decision-making. For instance, men and patients aged 70 and above may be advised to seek out medical consultation at an earlier stage of the disease, and when admission in hospital is required clinicians should be made aware of the higher risk of severe disease and mortality in these groups. For clinical trials and other human studies on COVID-19, in particular those evaluating possible treatments for COVID-19, it is especially important to control for age and sex as they are strong confounders.

\section{Limitations and strengths}

We should also consider some limitations. Most included studies, $\mathrm{n}=50$, were still from China involving Chinese patients with COVID-19 compared with $\mathrm{n}=9$ studies from outside China, potentially limiting the generalisability of the findings. Additional studies outside of China are expected and will be included in future updates of this living review. Additionally, the data extraction and quality assessment were performed by one reviewer. In future updates of this review, a second reviewer will (at least partially) reperform the data extraction.

Methodological limitations include the fact that disease severity was in most papers defined according to the clinical stages of COVID-19 issued by China and WHO interim guidance, ${ }^{7}$ but this was not always reported. Additionally, in some papers it was unclear whether severity was assessed on hospitalisation or during follow-up. This is additionally complicated by the fact that referral policy to dedicated hospitals in China obscures the severity on initial admission. Therefore, it was not always clear whether an RR or OR was the most appropriate risk measure. RRs were used to obtain conservative estimates.

Due to the observational design of the included studies, there may be confounding by differences in, for example, prehospitalisation health status and comorbidities. However, the observed differences in outcome for sex and age are consistent with other respiratory tract infections and there is a pathophysiological basis (eg, differences in immunity systems and response) that could explain the differences in outcome for sex and age that we observed.

Our review has the following strengths. Our search strategy was thorough and complete: we screened 11.550 individual records. After contacting corresponding authors, we were able to include additional data from 12 studies. The methodological quality as reflected by the NOS score was high and a thorough sensitivity analysis could not refute the conclusions. The possible influence of publication bias on our results was considered to be small: the time the included studies were published spans less than 4 months, almost all studies have a different research question than our questions and we were able to include extra (unpublished) data from 12 authors. This small influence of publication bias is confirmed by the small changes in effect size after the trim-and-fill analyses.
During the study selection phase we came across a number of studies that had to be excluded because of very short follow-up (days). As a consequence, the majority of included study subjects did not report on endpoints like recovery, discharge from hospital or mortality. Furthermore, information on the subjects without an endpoint was missing, so there was a high risk of non-differential misclassification that could lead to bias. For instance, in a particular study $20 \%$ had either recovered or diseased while $80 \%$ was still admitted in the hospital, and there was no information on the distribution of demographic factors for this $80 \%$. When confronted with these studies we contacted the authors and, in some cases, received information that allowed the study to be included.

\section{CONCLUSION}

We systematically reviewed the literature to describe the relation between age and sex and COVID-19 infection, disease severity, ICU admission and death. Meta-analyses on 59 studies comprising 36.470 patients showed that infection, severe disease, ICU admission and death are more likely to occur among men and patients aged 70 and above.

\section{Systematic review registration}

PROSPERO 2020: CRD42020180085 and online supplemental appendix 4 . Please note that we have prospectively reported when phases of the review started. However, these changes have not yet been made tothe online protocol. This delay in updates on the research protocol is probably due to the highworkload at Prospero.

\section{Author affiliations \\ ${ }^{1}$ Department of Orthopaedics, Leiden University Medical Center, Leiden, The Netherlands \\ ${ }^{2}$ Department of Methodology and Statistics, Care and Public Health Research Institute (CAPHRI), Maastricht University, Maastricht, The Netherlands \\ ${ }^{3}$ Department of Educational Research and Development, School of Health Professions Education, Maastricht University, Maastricht, The Netherlands ${ }^{4}$ Department of General Practice, Care and Public Health Research Institute (CAPHRI), Maastricht University, Maastricht, The Netherlands \\ ${ }^{5}$ Amsterdam University Medical Center, location VUmc, Amsterdam, The Netherlands ${ }^{6}$ Maastricht University Library, Maastricht University, Maastricht, The Netherlands \\ ${ }^{7}$ School of Mental Health and Neuroscience (MHeNS), Maastricht University, Maastricht, The Netherlands \\ ${ }^{8}$ Department of Research and Development, The Netherlands Comprehensive Cancer Organisation, Utrecht, The Netherlands \\ ${ }^{9}$ De Onderzoekerij, Leiden, The Netherlands \\ ${ }^{10}$ UCB Pharma B.V, Breda, The Netherlands \\ ${ }^{11}$ NUTRIM School of Translational Research in Metabolism, Care and Public Health Research Institute (CAPHRI), Maastricht University, Maastricht, The Netherlands}

Contributors MPZ conceived the study. All authors were involved in the study design during weekly meetings. GHLF designed and performed the search strategy. AVJ, RTD and BGP screened the titles and abstracts for eligibility. AR and SZ extracted the data (quantitative data) and AA, SH and JIRD reviewed the study quality (qualitative data). SJ analysed the data. BGP and SJ wrote the first draft. All authors revised this draft for critical content. All authors approved the final manuscript. MPZ, BGP and SJ are the guarantors. All persons listed as authors have contributed to preparing the manuscript. All authors had full access to all the data in the study and had final responsibility for the decision to submit for publication. 
Funding The authors have not declared a specific grant for this research from any funding agency in the public, commercial or not-for-profit sectors.

Dissemination declaration This review will be disseminated via WHO, direct communication with national centres for disease control, international library organisation and via Google Search engine optimisation provided by Maastricht University.

Competing interests None declared.

Patient consent for publication Not required.

Ethics approval For this systematic review and meta-analysis, approval by the ethics committee was not required.

Provenance and peer review Not commissioned; externally peer reviewed.

Data availability statement All data relevant to the study are included in the article or uploaded as supplemental information. The study protocol is available online at the PROSPERO website: https://www.crd.york.ac.uk/prospero/display_ record.php?RecordID=180085.

Supplemental material This content has been supplied by the author(s). It has not been vetted by BMJ Publishing Group Limited (BMJ) and may not have been peer-reviewed. Any opinions or recommendations discussed are solely those of the author(s) and are not endorsed by BMJ. BMJ disclaims all liability and responsibility arising from any reliance placed on the content. Where the content includes any translated material, BMJ does not warrant the accuracy and reliability of the translations (including but not limited to local regulations, clinical guidelines, terminology, drug names and drug dosages), and is not responsible for any error and/or omissions arising from translation and adaptation or otherwise.

Open access This is an open access article distributed in accordance with the Creative Commons Attribution Non Commercial (CC BY-NC 4.0) license, which permits others to distribute, remix, adapt, build upon this work non-commercially, and license their derivative works on different terms, provided the original work is properly cited, appropriate credit is given, any changes made indicated, and the use is non-commercial. See: http://creativecommons.org/licenses/by-nc/4.0/.

\section{ORCID iDs}

Bart G Pijls http://orcid.org/0000-0001-5351-5057

Anke Richters http://orcid.org/0000-0002-1580-1543

\section{REFERENCES}

1 Dong E, Du H, Gardner L. An interactive web-based dashboard to track COVID-19 in real time. Lancet Infect Dis 2020;20:533-4.

2 Falagas ME, Mourtzoukou EG, Vardakas KZ. Sex differences in the incidence and severity of respiratory tract infections. Respir Med 2007;101:1845-63.

3 Leung GM, Hedley AJ, Ho L-M, et al. The epidemiology of severe acute respiratory syndrome in the 2003 Hong Kong epidemic: an analysis of all 1755 patients. Ann Intern Med 2004;141:662-73.

4 Jin J-M, Bai P, He W, et al. Gender differences in patients with COVID-19: focus on severity and mortality. Front Public Health 2020;8:152.

5 Ferguson NM, Nedjati-Gilani G, Imai N. Report 9: impact of nonpharmaceutical interventions (NPIs) to reduce COVID-19 mortality and 392 healthcare demand. Imperial College COVID-19 Response Team 2020;10:77482.

6 Moher D, Liberati A, Tetzlaff J, et al. Preferred reporting items for systematic reviews and meta-analyses: the PRISMA statement. BMJ 2009;339:b2535.

7 World Health O. Clinical management of severe acute respiratory infection when novel coronavirus (2019-nCoV) infection is suspected: interim guidance, 28 January 2020. Geneva: World Health Organization, 2020.

8 NIHR Policy Research Programme Reviews Facility. COVID-19: a living systematic map of the evidence, 2020. Available: http://eppi. ioe.ac.uk/COVID19_MAP/covid_map_v5.html

9 Shea BJ, Reeves BC, Wells G, et al. AMSTAR 2: a critical appraisal tool for systematic reviews that include randomised or nonrandomised studies of healthcare interventions, or both. BMJ 2017;358:j4008.

10 et alWells G, Shea B, O'Connell D. The Newcastle-Ottawa Scale (NOS) for assessing the quality of nonrandomised studies in metaanalyses. 2013 [Available from:. Available: http://www.ohri.ca/ programs/clinical_epidemiology/oxford.asp

11 DerSimonian R, Laird N. Meta-Analysis in clinical trials. Control Clin Trials 1986;7:177-88.
12 Higgins JP, Thomas J, Chandler J. Cochrane Handbook for systematic reviews of interventions version 6.0 (updated July 2019).. Cochrane;2019.

13 Egger M, Smith GD, Schneider M, et al. Bias in meta-analysis detected by a simple, graphical test. BMJ 1997;315:629-34.

14 Harbord RM, Egger M, Sterne JAC. A modified test for small-study effects in meta-analyses of controlled trials with binary endpoints. Stat Med 2006;25:3443-57

$15 \mathrm{Ai}$, Yang Z, Hou H, et al. Correlation of chest CT and RT-PCR testing for coronavirus disease 2019 (COVID-19) in China: a report of 1014 cases. Radiology 2020;296:E32-40.

16 Cao J, Hu X, Cheng W, et al. Clinical features and short-term outcomes of 18 patients with corona virus disease 2019 in intensive care unit. Intensive Care Med 2020;46:851-3.

17 Chen G, Wu D, Guo W, et al. Clinical and immunological features of severe and moderate coronavirus disease 2019. J Clin Invest 2020;130:2620-9.

18 Chen Q, Zheng Z, Zhang C. Clinical characteristics of 145 patients with corona virus disease 2019 (COVID-19) in Taizhou, Zhejiang, China. Infection 2020:1-9 (published Online First: 2020/04/29).

19 Chen R, Liang W, Jiang M, et al. Risk Factors of Fatal Outcome in Hospitalized Subjects With Coronavirus Disease 2019 From a Nationwide Analysis in China. Chest 2020;158:97-105.

20 Chen T, Dai Z, Mo P, et al. Clinical characteristics and outcomes of older patients with coronavirus disease 2019 (COVID-19) in Wuhan, China: a Single-Centered, retrospective study. J Gerontol A Biol Sci Med Sci 2020;75:1788-95.

21 Chen X, Zhao B, Qu Y, et al. Detectable serum severe acute respiratory syndrome coronavirus 2 viral load (RNAemia) is closely correlated with drastically elevated interleukin 6 level in critically ill patients with coronavirus disease 2019. Clin Infect Dis 2020;71:1937-42.

22 Chu J, Yang N, Wei Y, et al. Clinical characteristics of 54 medical staff with COVID-19: a retrospective study in a single center in Wuhan, China. J Med Virol 2020;92:807-13.

23 Colaneri M, Sacchi P, Zuccaro V, et al. Clinical characteristics of coronavirus disease (COVID-19) early findings from a teaching hospital in Pavia, North Italy, 21 to 28 February 2020. Euro Surveill 2020;25:2000460.

24 Dong Y, Mo X, Hu Y, et al. Epidemiology of COVID-19 among children in China. Pediatrics 2020;145:e20200702.

25 Du R-H, Liang L-R, Yang C-Q, et al. Predictors of mortality for patients with COVID-19 pneumonia caused by SARS-CoV-2: a prospective cohort study. Eur Respir J 2020;55:2000524.

26 Fan BE, Chong VCL, Chan SSW, et al. Hematologic parameters in patients with COVID-19 infection. Am J Hematol 2020;95:E131-4.

27 Gao Y, Li T, Han M, et al. Diagnostic utility of clinical laboratory data determinations for patients with the severe COVID-19. J Med Virol 2020;92:791-6.

28 Guan W-jie, Ni Z-yi, Hu Y, et al. Clinical characteristics of coronavirus disease 2019 in China. N Engl J Med Overseas Ed 2020;382:1708-20.

29 Huang C, Wang Y, Li X, et al. Clinical features of patients infected with 2019 novel coronavirus in Wuhan, China. The Lancet 2020;395:497-506

30 Kalligeros M, Shehadeh F, Mylona EK, et al. Association of obesity with disease severity among patients with coronavirus disease 2019 . Obesity 2020;28:1200-4.

31 Li K, Wu J, Wu F, et al. The clinical and chest CT features associated with severe and critical COVID-19 pneumonia. Invest Radiol 2020;55:327-31

32 Liu R, Han H, Liu F, et al. Positive rate of RT-PCR detection of SARSCoV-2 infection in 4880 cases from one hospital in Wuhan, China, from Jan to Feb 2020. Clinica Chimica Acta 2020;505:172-5.

33 Liu $\mathrm{R}, \mathrm{Ma} \mathrm{Q}$, Han $\mathrm{H}$, et al. The value of urine biochemical parameters in the prediction of the severity of coronavirus disease 2019. Clin Chem Lab Med 2020;58:1121-4.

34 Liu Y, Mao B, Liang S, et al. Association between age and clinical characteristics and outcomes of COVID-19. Eur Respir J 2020;55:2001112.

35 Long $\mathrm{C}, \mathrm{Xu} \mathrm{H}$, Shen $\mathrm{Q}$, et al. Diagnosis of the coronavirus disease (COVID-19): rRT-PCR or CT? Eur J Radiol 2020;126:108961.

36 Long L, Zeng X, Zhang X, et al. Short-term outcomes of COVID-19 and risk factors for progression. Eur Respir J 2020;55. doi:10.1183/13993003.00990-2020. [Epub ahead of print: 2705 2020].

37 Lyu P, Liu X, Zhang R, et al. The performance of chest CT in evaluating the clinical severity of COVID-19 pneumonia: identifying critical cases based on CT characteristics. Invest Radiol 2020:55:412-21. 
38 Meng Y, Wu P, Lu W, et al. Sex-specific clinical characteristics and prognosis of coronavirus disease-19 infection in Wuhan, China: a retrospective study of 168 severe patients. PLoS Pathog 2020:16:e1008520.

39 Myers LC, Parodi SM, Escobar GJ, et al. Characteristics of hospitalized adults with COVID-19 in an integrated health care system in California. JAMA 2020;323:2195-8.

40 Nikpouraghdam M, Jalali Farahani A, Alishiri G, et al. Epidemiological characteristics of coronavirus disease 2019 (COVID-19) patients in Iran: a single center study. J Clin Virol 2020;127:104378.

41 Pei G, Zhang Z, Peng J, et al. Renal involvement and early prognosis in patients with COVID-19 pneumonia. JASN 2020;31:1157-65.

42 Qian G-Q, Yang N-B, Ding F, et al. Epidemiologic and clinical characteristics of 91 hospitalized patients with COVID-19 in Zhejiang, China: a retrospective, multi-centre case series. QJM 2020;113:474-81.

43 Qin C, Zhou L, Hu Z, et al. Dysregulation of immune response in patients with coronavirus 2019 (COVID-19) in Wuhan, China. Clin Infect Dis 2020;71:762-768.

44 Richardson S, Hirsch JS, Narasimhan M, et al. Presenting characteristics, comorbidities, and outcomes among 5700 patients hospitalized with COVID-19 in the new York City area. JAMA 2020;323:2052-9.

45 Rieg S, Busch H-J, Hans F, et al. [COVID-19-response strategies of the task-force Coronavirus and experiences upon implementation in the management of 115 cases at the University Medical Center Freiburg]. Dtsch Med Wochenschr 2020;145:657-64.

46 Shen N, Zhu Y, Wang X, et al. Characteristics and diagnosis rate of 5630 subjects receiving SARS-CoV-2 nucleic acid tests from Wuhan, China. JCl Insight 2020;5.

47 Shi Y, Yu X, Zhao H, et al. Host susceptibility to severe COVID-19 and establishment of a host risk score: findings of 487 cases outside Wuhan. Crit Care 2020;24:108.

48 Tang N, Li D, Wang X, et al. Abnormal coagulation parameters are associated with poor prognosis in patients with novel coronavirus pneumonia. J Thromb Haemost 2020;18:844-7.

49 COVID-19 National Emergency Response Center, Epidemiology and Case Management Team, Korea Centers for Disease Control and Prevention. Coronavirus Disease-19: summary of 2,370 contact investigations of the first 30 cases in the Republic of Korea. Osong Public Health Res Perspect 2020;11:81-4.

50 Tian S, Hu N, Lou J, et al. Characteristics of COVID-19 infection in Beijing. J Infect 2020;80:401-6.

51 Tomlins J, Hamilton F, Gunning S, et al. Clinical features of 95 sequential hospitalised patients with novel coronavirus 2019 disease (COVID-19), the first UK cohort. J Infect 2020;81:e59-61.

52 Wan S, Xiang Y, Fang W, et al. Clinical features and treatment of COVID-19 patients in northeast Chongqing. J Med Virol 2020;92:797-806.

53 Wang D, Hu B, Hu C, et al. Clinical characteristics of 138 hospitalized patients with 2019 novel Coronavirus-Infected pneumonia in Wuhan, China. JAMA 2020;323:1061-9.

54 Wang $\mathrm{L}, \mathrm{Li}$ X, Chen $\mathrm{H}$, et al. Coronavirus disease 19 infection does not result in acute kidney injury: an analysis of 116 hospitalized patients from Wuhan, China. Am J Nephrol 2020;51:343-8.

55 Wang R, Pan M, Zhang X, et al. Epidemiological and clinical features of 125 hospitalized patients with COVID-19 in Fuyang, Anhui, China. Int J Infect Dis 2020;95:421-8.

56 Wang X, Fang J, Zhu Y, et al. Clinical characteristics of non-critically ill patients with novel coronavirus infection (COVID-19) in a Fangcang Hospital. Clin Microbiol Infect 2020;26:1063-8.

57 Wei J-F, Huang F-Y, Xiong T-Y, et al. Acute myocardial injury is common in patients with COVID-19 and impairs their prognosis. Heart 2020;106:1154-9.

$58 \mathrm{Wu} \mathrm{C}$, Chen X, Cai Y, et al. Risk factors associated with acute respiratory distress syndrome and death in patients with coronavirus disease 2019 pneumonia in Wuhan, China. JAMA Intern Med 2020;180:934.

$59 \mathrm{Wu}$ J, Li W, Shi X, et al. Early antiviral treatment contributes to alleviate the severity and improve the prognosis of patients with novel coronavirus disease (COVID-19). J Intern Med 2020;288:128-38.

60 Xu B, Fan C-yu, Wang A-lu, et al. Suppressed T cell-mediated immunity in patients with COVID-19: a clinical retrospective study in Wuhan, China. Journal of Infection 2020;81:e51-60.
61 Yan Y, Yang Y, Wang F, et al. Clinical characteristics and outcomes of patients with severe covid-19 with diabetes. BMJ Open Diab Res Care 2020;8:e001343.

62 Yang R, Gui X, Zhang Y, et al. The role of essential organ-based comorbidities in the prognosis of COVID-19 infection patients. Expert Rev Respir Med 2020;14:1-4.

63 YuX, Sun S, Shi Y, et al. SARS-CoV-2 viral load in sputum correlates with risk of COVID-19 progression. Crit Care 2020;24:170.

64 Zhang G, Hu C, Luo L, et al. Clinical features and short-term outcomes of 221 patients with COVID-19 in Wuhan, China. J Clin Virol 2020;127:104364

65 Zhang G, Zhang J, Wang B, et al. Analysis of clinical characteristics and laboratory findings of 95 cases of 2019 novel coronavirus pneumonia in Wuhan, China: a retrospective analysis. Respir Res 2020;21:74

66 Zhang J, Wang X, Jia X, et al. Risk factors for disease severity, unimprovement, and mortality in COVID-19 patients in Wuhan, China. Clin Microbiol Infect 2020;26:767-72.

67 Zhang J-J, Dong X, Cao Y-Y, et al. Clinical characteristics of 140 patients infected with SARS-CoV-2 in Wuhan, China. Allergy 2020;75:1730-41.

68 Zhang R, Ouyang H, Fu L, et al. Ct features of SARS-CoV-2 pneumonia according to clinical presentation: a retrospective analysis of 120 consecutive patients from Wuhan City. Eur Radiol 2020;30:4417-26.

69 Zhao X-Y, Xu X-X, Yin H-S, et al. Clinical characteristics of patients with 2019 coronavirus disease in a non-Wuhan area of Hubei Province, China: a retrospective study. BMC Infect Dis 2020;20:311.

70 Zheng F, Tang W, Li H, et al. Clinical characteristics of 161 cases of corona virus disease 2019 (COVID-19) in Changsha. Eur Rev Med Pharmacol Sci 2020;24:3404-10.

71 Zheng S, Fan J, Yu F. Viral load dynamics and disease severity in patients infected with SARS-CoV-2 in Zhejiang Province, China, January-March 2020: retrospective cohort study. BMJ;2020:m1443.

72 Zhu W, Xie K, Lu H, et al. Initial clinical features of suspected coronavirus disease 2019 in two emergency departments outside of Hubei, China. J Med Virol 2020;92:1525-32.

73 Zhu Z, Cai T, Fan L, et al. Clinical value of immune-inflammatory parameters to assess the severity of coronavirus disease 2019. Int $J$ Infect Dis 2020:95:332-9.

$74 \varnothing \mathrm{E}$, Brurberg KG, Nytrøen K. Rapid methods including network meta-analysis to produce evidence in clinical decision support: a decision analysis. Syst Rev 2018;7:168.

75 Wynants L, Van Calster B, Collins GS. Prediction models for diagnosis and prognosis of covid-19 infection: systematic review and critical appraisal. BMJ 2020;369:m1328.

76 Elliott $\mathrm{JH}$, Turner T, Clavisi O, et al. Living systematic reviews: an emerging opportunity to narrow the evidence-practice gap. PLoS Med 2014;11:e1001603.

77 Vandvik PO, Brignardello-Petersen R, Guyatt GH. Living cumulative network meta-analysis to reduce waste in research: a paradigmatic shift for systematic reviews? BMC Med 2016;14:59.

78 Beenakker KGM, Westendorp RGJ, de Craen AJM, et al. Men have a stronger monocyte-derived cytokine production response upon stimulation with the gram-negative stimulus lipopolysaccharide than women: a pooled analysis including 15 study populations. J Innate Immun 2020;12:142-53.

79 Klein SL, Flanagan KL. Sex differences in immune responses. Nat Rev Immunol 2016;16:626-38.

80 Aziz M, Fatima R, Assaly R. Elevated interleukin-6 and severe COVID-19: a meta-analysis. J Med Virol 2020;92:2283-5.

81 Jamilloux $Y$, Henry T, Belot A, et al. Should we stimulate or suppress immune responses in COVID-19? cytokine and anti-cytokine interventions. Autoimmun Rev 2020;19:102567.

82 Mörs K, Braun O, Wagner N, et al. Influence of gender on systemic IL-6 levels, complication rates and outcome after major trauma. Immunobiology 2016;221:904-10.

83 Qiao Z, Wang W, Yin L, et al. Using IL-6 concentrations in the first $24 \mathrm{~h}$ following trauma to predict immunological complications and mortality in trauma patients: a meta-analysis. Eur J Trauma Emerg Surg 2018;44:679-87.

84 Shaw AC, Joshi S, Greenwood H, et al. Aging of the innate immune system. Curr Opin Immunol 2010;22:507-13.

85 Licastro F, Candore G, Lio D, et al. Innate immunity and inflammation in ageing: a key for understanding age-related diseases. Immun Ageing 2005;2:8. 